
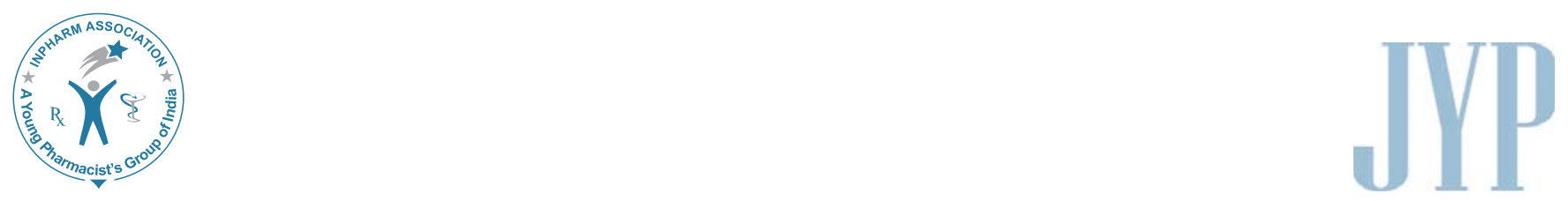

\title{
Gene Polymorphism in Amlodipine Induced Gingival Hyperplasia: A Case Report
}

\author{
Charles N, Ramesh V, Kennedy Babu SPK, Premalatha B \\ Department of Oral Pathology and Microbiology, Mahatma Gandhi Postgraduate Institute of Dental \\ Sciences, Indira Nagar, Gorimedu, Puducherry, India \\ Address for correspondence: Dr. Noronha Shyam Curtis Charles; E-mail: amitojsall@gmail.com
}

\begin{abstract}
The management of cardiovascular disorders poses a dilemma for the medical fraternity. Calcium channel blockers are one of the most commonly used drugs for the management of this disorder, and it is also a well known fact that they are one of the most common group of drugs responsible for causing gingival over growth as one of their adverse effects. Amlodipine is a new generation hypertensive, which has found wide acceptance and usage due to its duration of action. Even with all its benefits as a potent hypertensive, its effect on gingival tissues is what causes concern to the patient and dental surgeon equally. The objective of this article is to create awareness regarding the adverse oral effects of amlodipine, its underlying mechanism of action in bringing about this adverse reaction, along with providing a brief review of the pharmacologic profile of this drug.
\end{abstract}

Key words: Gingival hyperplasia, amlodipine, gingival pocket

\section{INTRODUCTION}

Gingival hyperplasia is a serious concern both for the patient and the clinician. The main factors which may be responsible for gingival hyperplasia are inflammation and fibrosis, or a combination of both. A number of local and systemic conditions have been attributed as causative agents of generalized gingival inflammatory enlargement. However, fibrous enlargement is mainly due to idiopathic gingival fibromatosis or drug-induced.

\begin{tabular}{|l|l|}
\hline \multicolumn{2}{|c|}{ Access this article online } \\
\hline Quick Response Code: & \\
\hline & Website: \\
\hline
\end{tabular}

Anticonvulsants, antihypertensive calcium antagonists, and the immunosuppressant cyclosporine are the main classes of drugs known to cause gingival hyperplasia. The pharmacologic effect of each of these drugs is different but all of them seem to act similarly on secondary target tissue, i.e. the gingival connective tissue, thereby resulting in common clinical histopathological findings. Calcium antagonists are widely used in medical practice for the management of cardiovascular disorders. Amongst this large group of drugs, the dihydropyridines are the agents most frequently implicated in gingival enlargement. Amlodipine has rarely been reported as the potential etiologic cause of gingival hyperplasia. ${ }^{[1,2]} \mathrm{A}$ rare case of amlodipine-induced gingival hyperplasia in tertiary care hospital is described with the evidence of pharmacogenetic analysis.

A 60 year-old female patient reported to the Department 
of Periodontics, Mahatma Gandhi Post Graduate Institute of Dental Sciences, Puducherry complaining of "swollen gums". Past medical history revealed that the patient had been diagnosed with hypertension 3 years back for which amlodipine ( $05 \mathrm{mg}$ /day, single dose orally) was prescribed for the last 3 years. The patient had noted a gradual, painless enlargement of the gingiva for the past 10 days. Clinical examination revealed a generalized and firm overgrowth of the gingiva throughout the maxilla and mandible involving the interdental, attached and marginal gingival [Figures 1 and 2]. On probing of periodontal pockets (mean probing depth: $2.3 \mathrm{~mm}$, SD: 1.1) were obvious which was a prominent feature of gingival overgrowth indicating an outward rather than vertical enlargement of gingiva. No changes in the gingival color or purulent discharge were noticed. Bleeding on probing was detected which was in accordance with mild superimposed gingival inflammation. Histopathologic inspection of the gingival biopsy specimens revealed connective tissue hyperplasia, acanthosis of overlying epithelium, and elongated rete ridges together with few sparse inflammatory cells in the sub epithelial area. The lesion was diagnosed as amlodipineinduced gingival hyperplasia based on history, clinical and histopathological findings [Figure 3].

Five milliliters of blood sample was collected from subject's antecubital veinin a polypropylene tube containing $100 \mu \mathrm{l}$ of anticoagulant $(10 \%$ ethylene diaminetetraacetic acid (EDTA). Phenol-chloroform method was used to extract the genomic DNA. The extracted DNA was diluted to $50 \mathrm{ng} / \mu \mathrm{l}$ concentration and was stored at $-20^{\circ} \mathrm{C}$. The sample was genotyped for MDR1 $3435 C>$ T using custom TaqMan genotyping assay on a real-time thermocycler by allelic discrimination method (Applied Biosystemsreal time thermocycler 7300, Foster City, CA) and the same methodology was validated by performing direct gene sequencing. The genotype obtained for MDR1 (3435C > T) polymorphism was a heteromutant (CT) genotype.

Currently, the etiology of drug-induced gingival overgrowth is not entirely understood, but it has now become quite clear that a multifactorial role may be involved in its cause. The controversy of the fact whether drug-induced gingival overgrowth is due to hyperplasia of the gingival epithelium or of sub mucosal connective tissue, and/or both still remains an enigma. Furthermore, the effect of age, sex, duration and dosage of the drug in the pathogenesis of gingival overgrowth is not clearly understood. Some of the risk factors known to contribute the gingival overgrowth include, presence of gingival inflammation resulting from poor oral hygiene. Furthermore, the presence of dental plaque may provide a reservoir for the accumulation of drugs causing gingival enlargement such as amlodipine. Other intrinsic risk factors that increase the susceptibility

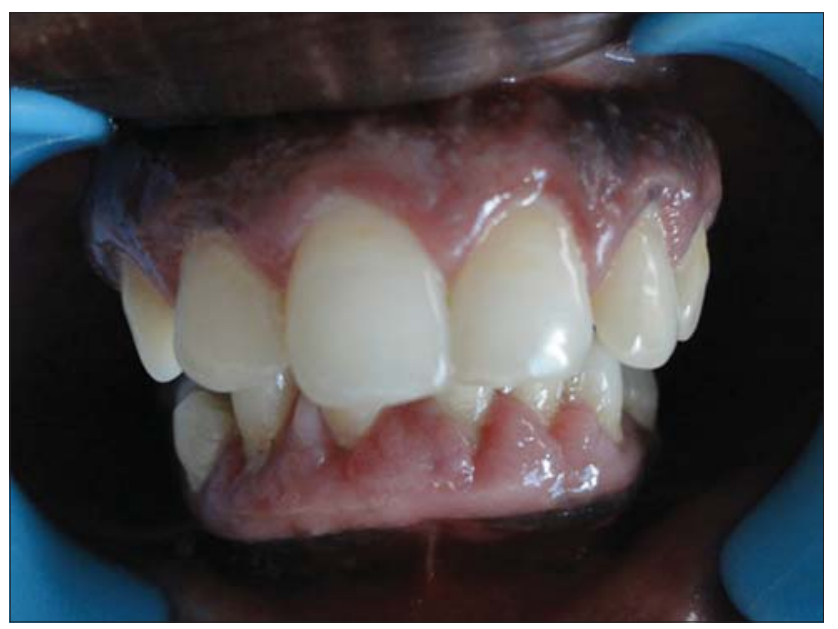

Figure 1: Diffuse gingival enlargement seen on the labial surface of maxillary and mandibular gingival

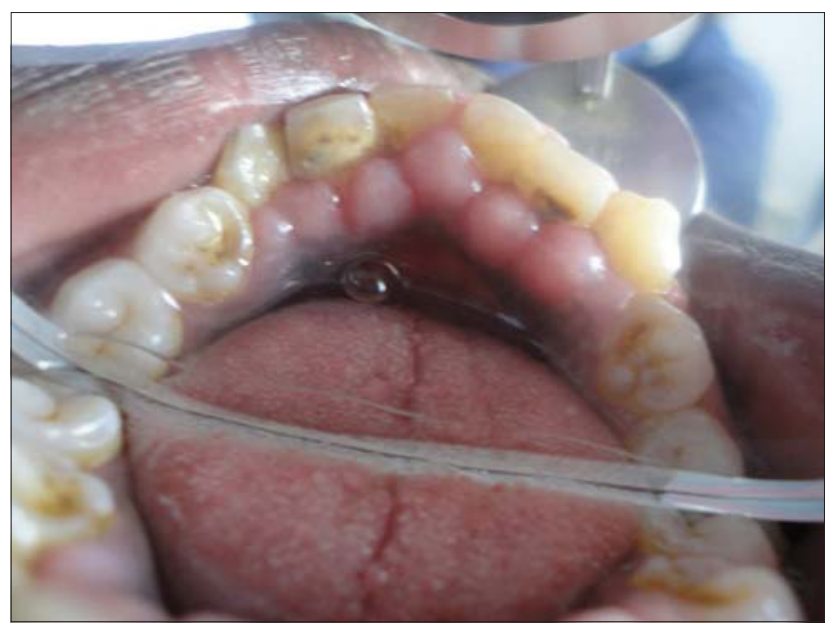

Figure 2: Gingival enlargement of interdental gingival seen on the lingual surface of mandibular gingival

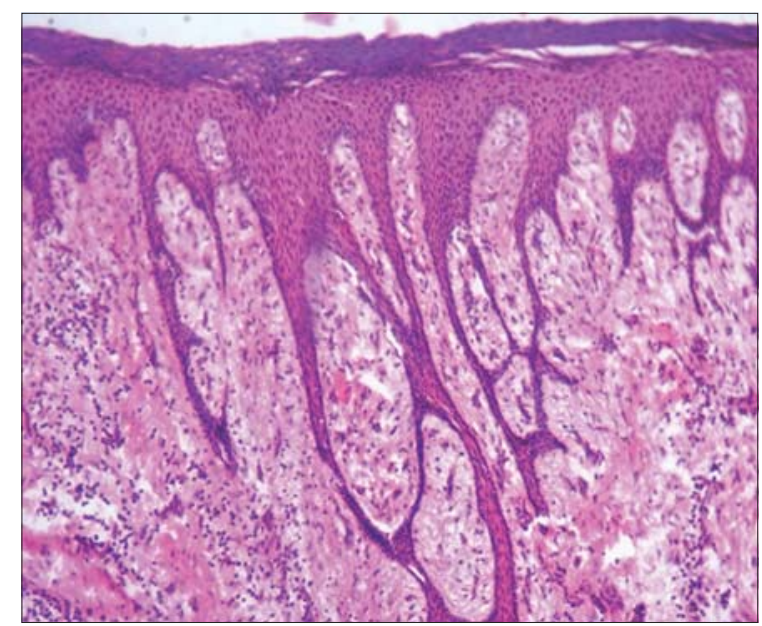

Figure 3: Photomicrograph showing connective tissue hyperplasia, acanthosis of overlying epithelium, and elongated rete ridges together with few sparse inflammatory cells in the subepithelial area (10x) 
of patients to drug induced gingival overgrowth are the fibroblasts which have been thought to have an abnormal vulnerability to the drug in affected individuals. It has been proven experimentally that fibroblast from drug induced hyperplasic gingiva in these patients show an increased level of collagen synthesis. It has been hypothesized that vulnerability or resistance to drug induced gingival enlargement may be caused by the existence of variable proportions of fibroblast subsets in each individual thus eliciting a fibrogenic response. ${ }^{[3,4]}$ As far as the role of inflammatory cytokines is concerned, it was proven that when human gingival fibroblasts were simultaneously exposed to nifedipine and pro-inflammatory cytokines (interleukin-1b and IL-6), that are elevated in inflamed gingival tissues, an up regulation of synthesis of collagen was observed. ${ }^{[4,5]}$ It has also been postulated that matrix metalloproteinases (MMPs) which are implicated in gingival enlargement may interfere with the synthesis and function of collagenases. This hypothesis was based on their negative effects on calcium ion influx across cell membranes. Furthermore, as gingival overgrowth is known to occur as an adverse drug reaction of calcium antagonists, studies carried out shows a modulation of inflammatory processes. As the calcium antagonists act as inhibitors of P-glycoprotein (P-gp) to a variable degree, the genetic product of Multidrug Resistance1 (MDR1) and inflammation may modify the P-gp expression, which is expressed in the endothelial layers of blood vessels obtained from healthy or inflamed gingiva. It is also found that deeper gingival pockets/pseudo pockets existed in subjects treated with calcium antagonists (Amlodipine), as compared to drug free counterparts. It has been found that this drug related side effect is associated with the MDR1 $3435 \mathrm{C} / \mathrm{T}$ gene polymorphism.
The reported case is an example of a combined type of gingival enlargement; basically drug induced, complicated by inflammatory changes due to plaque accumulation. Among the overall pharmacologic agents involved in gingival enlargement, phenytoin has the highest prevalence rate (approximately 50\%), with calcium channel blockers and cyclosporine associated enlargements about half as prevalent. In this particular case, treatment with calcium antagonists namely amlodipine has lead to gingival hyperplasia associated with MDR1 $3435 \mathrm{C} / \mathrm{T}$ polymorphism. The MDR1 polymorphism may modify the inflammatory response to the drug. If possible, treatment is generally targeted on drug substitution and effective control of local inflammatory factors by preventing plaque and calculus formation. When these measures fail to cause resolution of the enlargement, surgical intervention is recommended.

\section{REFERENCES}

1. Eggerath J, English H, Leichter JW. Drug-associated gingival enlargement: Case report and review of aetiology, management and evidence-based outcomes of treatment. J N Z Soc Periodontol 2005;88:7-14.

2. Jorgensen MG. Prevalence of Amlodipine-related gingival hyperplasia. J Periodontol 1997;68:676-8.

3. Johnson RB, Zebrowski EJ, Dai X. Synergistic enhancement of college nous protein synthesis by human gingival fibroblasts exposed to nifedipine and interleukin-1-beta in vitro. J Oral Pathol Med 2000;29:8-12.

4. Grover V. Amlodipine induced gingival hyperplasia. J Oral Health Comm Dent 2007;1:19-22.

5. Williamson MS, Miller EK, Plemons J, Rees T, Lacopino AM. Cyclosporine Aupregulates interleukin-6 gene expression in human gingival: Possible mechanism for gingival overgrowth. J Periodontol1994;11:552-60.

How to cite this article: Charles N, Ramesh V, Kennedy Babu SPK, Premalatha B. Gene polymorphism in amlodipine induced gingival hyperplasia: A case report. J Young Pharmacists 2012;4:287-9.

Source of Support: Nil, Conflict of Interest: None declared. 